This item was submitted to Loughborough's Research Repository by the author.

Items in Figshare are protected by copyright, with all rights reserved, unless otherwise indicated.

\title{
An actively constrained two degree-of-freedom manipulator for passive deployment applications
}

PLEASE CITE THE PUBLISHED VERSION

PUBLISHER

Professional Engineering Publishing / @ IMECHE

VERSION

VoR (Version of Record)

LICENCE

CC BY-NC-ND 4.0

\section{REPOSITORY RECORD}

Reedman, Adam V.C., and Kaddour Bouazza-Marouf. 2019. "An Actively Constrained Two Degree-of-freedom Manipulator for Passive Deployment Applications”. figshare. https://hdl.handle.net/2134/5139. 
This item was submitted to Loughborough's Institutional Repository (https://dspace.lboro.ac.uk/) by the author and is made available under the following Creative Commons Licence conditions.

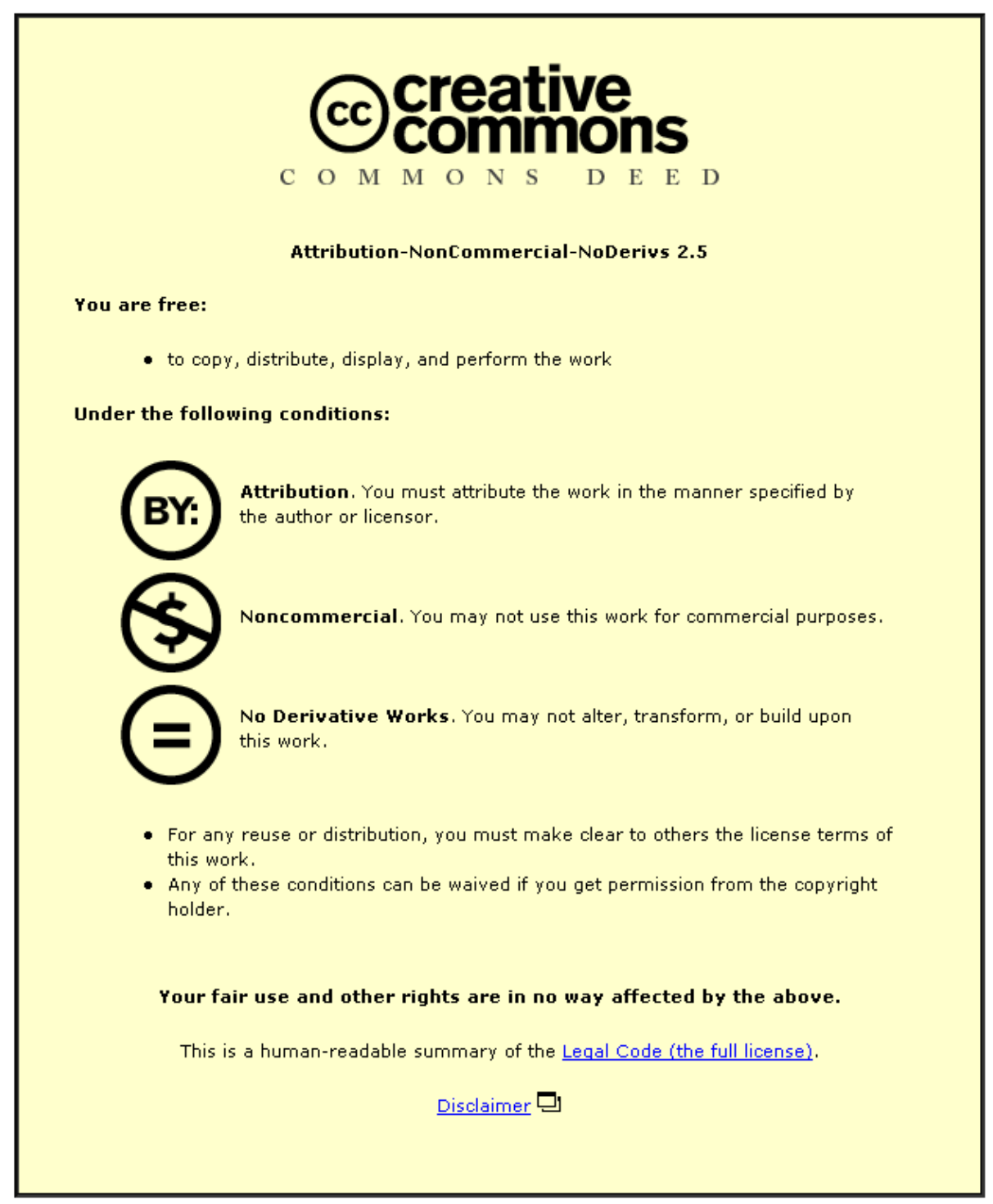

For the full text of this licence, please go to: http://creativecommons.org/licenses/by-nc-nd/2.5/ 


\title{
An actively constrained two degree-of-freedom manipulator for passive deployment applications
}

\author{
A V C Reedman and K Bouazza-Marouf* \\ Wolfson School of Mechanical and Manufacturing Engineering, Loughborough University, Loughborough, \\ Leicestershire, UK
}

\begin{abstract}
A two degree-of-freedom manipulator using actively constrained revolute joints is presented in this paper. Each revolute joint drive mechanism consists of two motor-driven worms coupled to a single worm wheel. A mathematical model of the manipulator system is used in order to develop a computed-torque control algorithm. Experimental results show that the position of the end-effector can be successfully controlled to track a path generated from a user-input force command signal while cancelling backlash at the gear interface. This system has been designed for the purpose of following a predefined path under the direct physical control of the user. The joint mechanism and control strategy used in this paper allow for backlash to be continuously cancelled. The safety of the user is ensured by enabling joint motions only if a user force is applied, and this force is in a direction that allows the controller to restrict joint motion along a predefined path.
\end{abstract}

Keywords: robotics, computed-torque control, passive deployment

\section{NOTATION}

$a_{n}$

B

$B_{i j}$

C

$C_{i j}$

$\mathrm{C}_{\mathrm{r}}$

$\mathrm{C}_{r 11}$

$\boldsymbol{F}_{\mathrm{u}}$

$F_{\text {u,max }}$

$F_{\text {u,min }}$

$\mathbf{J}$

$\bar{J}_{n}$

$J_{\theta n}$

$K_{\mathrm{d} \alpha n}, K_{\mathrm{i} \alpha n}, K_{\mathrm{p} \alpha n}$ position of the centre of mass of

link $n$

torque conversion matrix

element $i j$ of $\mathbf{B}$

manipulator viscous damping matrix

element $i j$ of $\mathbf{C}$

referred manipulator viscous damping

matrix from worm mechanisms

element $i j$ of $\mathbf{C}_{\mathrm{r}}$

user-input force

user-input force at which the maximum end-effector speed is reached

minimum user-input force allowed to generate motion

manipulator arm Jacobian matrix

mass moment of inertia about the

centre of mass of link $n$

residual inertia of link $n$

derivative, integral and proportional controller gains for $\alpha$ unwinding of joint $n$
$K_{\mathrm{d} \beta n}, K_{\mathrm{i} \beta n}, K_{\mathrm{p} \beta n}$

\section{$l_{n}$}

$m_{n}$

M

$\mathbf{M}_{\mathrm{r}}$

$\mathbf{M}_{\theta}$

$q$

$q_{\mathrm{d}}$

$r_{\alpha n}$

$r_{\beta n}$

$r_{\theta n}$

$V_{\mathrm{d}, \max }$

$V_{\mathrm{d}, \min }$

$\mathbf{V}_{\mathrm{m}}$

$\gamma_{n}$

$\varepsilon_{n}$

$\theta_{n}$

$\theta_{n \mathrm{~d}}$ derivative, integral and proportional controller gains for $\beta$ unwinding of joint $n$

length of link $n$

mass of link $n$

manipulator inertia matrix

referred inertia matrix from worm mechanisms

residual inertia matrix

vector of joint positions

vector of desired joint positions

radius of contact of $\alpha$ worm with worm wheel for link $n$

radius of contact of $\beta$ worm with worm wheel for link $n$

radius of contact of worm wheel with $\alpha$ and $\beta$ worms for link $n$ maximum allowable speed of the end-effector

minimum allowable speed of the end-effector matrix of Coriolis and centrifugal terms

worm lead angle of joint $n$ friction constant for joint $n$ position of joint $n$ desired position of joint $n$ 


$\begin{array}{ll}\mu_{\alpha n}, \mu_{\mathrm{d} \beta n} & \begin{array}{l}\text { coefficient of dynamic friction of } \\ \alpha \text { and } \beta \text { worms of joint } n\end{array} \\ \mu_{\mathrm{s} \alpha n}, \mu_{\mathrm{s} \beta n} & \begin{array}{l}\text { coefficient of static friction of } \alpha \text { and } \\ \beta \text { worms of joint } n\end{array} \\ \boldsymbol{\tau} & \begin{array}{l}\text { joint actuator torque vector } \\ \boldsymbol{\tau}_{\mathrm{act}}\end{array} \\ & \begin{array}{l}\text { vector of joint torques obtained from } \\ \text { strain gauges measurements }\end{array} \\ \tau_{\mathrm{act}, n} & \text { measured torques of joint } n \\ \boldsymbol{\tau}_{\mathrm{m}} & \text { vector of } \alpha \text { and } \beta \text { motor torques } \\ \tau_{\mathrm{m} \alpha n}, \tau_{\mathrm{m} \beta n} & \alpha \text { and } \beta \text { motor torques of joint } n\end{array}$

\section{INTRODUCTION}

Development of manipulators that interact closely with humans is increasingly a focus of research in the field of robotics. In particular, robotic devices have been introduced into the operating theatre to help the surgeon perform intricate invasive procedures with a higher degree of accuracy and reliability than would otherwise be possible with more traditional methods.

A wide variety of operations have benefited from the introduction of a robotic assistant. For example, orthopaedic, neurological, cardiac and cosmetic surgical procedures have all been enhanced by robotic assistance. However, many of the robotic devices currently in use are based on modified industrial manipulators which have typically been designed to meet the requirements of high-speed and high-torque applications. The accuracy of such devices is unsurpassed but in such safety-critical environments the use of high-powered mechanisms to perform these tasks has the potential to compromise the safety of patient, surgeon and operating room staff.

Without very expensive and necessary software and hardware safety features, these robots cannot be allowed to operate in safety-critical environments such as an operating theatre. As a result more recent research has focused on designing semi-active and passive devices that are designed to mechanically remove the potential dangers posed by high-powered manipulators. In particular, passive arms with dynamic constraints have been identified as a safe alternative to modified industrial manipulators. Robots such as PADyC (passive arm with dynamic constraints) [1], PTER (passive trajectory enhancing robot) [2] and COBOT (collaborative robot) [3] have been used to demonstrate that computer controlled clutches and brakes can limit the range of motion of the endeffector to remain within a predefined safe working region. Although these devices have proved relatively successful in accomplishing this task, they have had only limited success in constraining the tool (i.e. the end-effector) to remain on a predefined path, as may be required by more complex machining processes such as bone milling and drilling involved in modern surgical procedures.

In this paper, a two degree-of-freedom (2 DOF) revolute joint planar manipulator is presented that is suitable for use in applications where direct control of the end-effector by a human is required. The robot is developed based on the mechanical and control principles given in a previous paper [4]. A conceptual overview of the system is given and a mathematical model of the 2 DOF system is used to develop a computed-torque control algorithm for trajectory tracking. A novel pointer mechanism used in conjunction with the control strategies to help the user to complete the desired task is presented. A description of the experimental set-up and control system architecture is also included. Experimental results using the computedtorque control algorithm to guide the end-effector around a circular path under direct user control are presented, followed by concluding remarks.

\section{CONCEPTUAL OVERVIEW}

\subsection{Dual-worm driven joint}

Each joint, shown schematically in Fig. 1, consists of two worms, each driven by a low-power d.c. servomotor. The two worms follow a worm wheel that is fixed to the robot link. The inability of the worm mechanism to be back-driven means that no matter how much force the operator applies, motion is not allowed until both motors are controlled to move in a positive or negative direction. The dual-worm mechanism also has the ability to eliminate backlash using a control strategy that is discussed below.

The parallel nature of this overactuated joint structure makes this active system safer than modified industrial robots. Should there be a failure, both motors would have to drive in opposite directions to achieve motion.

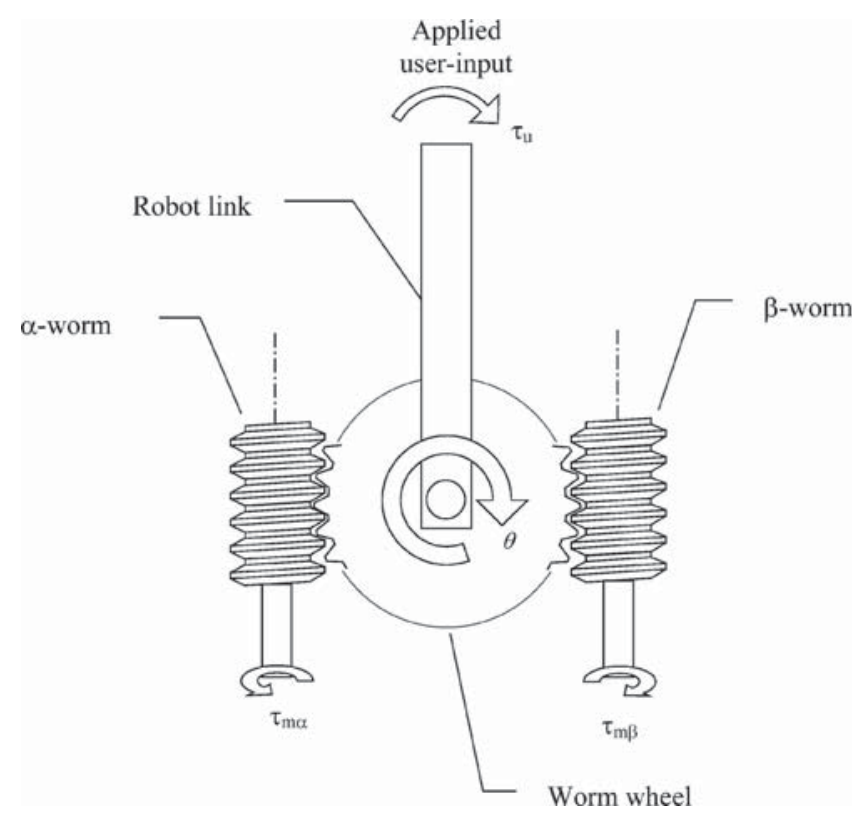

Fig. 1 Single DOF dual-worm drive joint 
However, if this failure occurs, speed of the joints would be very small, giving the operator plenty of time to react, i.e. to release the dead-man's-handle. The motors were selected to give a peak joint speed of $2 \mathrm{r} / \mathrm{min}$.

\subsection{Two DOF manipulator}

The proposed system is intended for medical/surgical applications. Many surgical assistant systems use computer graphics to convey information to the surgeon. However, this can be distracting because of the need, for the surgeon, to be able to focus not only on the task in hand but also on the monitor that is giving directions. The use of a graphical interface in this manner requires the surgeon to transform the directions from the information provided on the screen to the real-world robot. This may not always be easy or possible and would increase the time required to complete a task, contradicting the purpose of developing the manipulator in the first instance. Extensive training of the user in the operation of the system may also be required. The use of revolute joint architectures for passive deployment applications has been shown to be a significant problem. In particular, the PADyC system requires the user to 'feel' his or her way along a narrow corridor that describes the actual desired path. Sudden changes in the system dynamics caused by the clutches have to be compensated for by the user. This may require training of the user in the use of the system. Revolute joint devices such as PADyC, which rely on the user 'feeling' his or her way by applying a force in an appropriate direction, have clearly identified that it is not sufficient to simply show the user what the task is by, for example, displaying the path on a computer screen. It should be noted that motion of the end-effector along a particular path is possible only if the torque applied on each joint, as a result of the user force, produces motion (i.e. rotation) of the corresponding joint in the desired direction. This is explained below using a random kinematic configuration of a 2 DOF system.

Consider a 2 DOF revolute joint manipulator as shown in Fig. 2a that is required to follow a predefined path, $\mathrm{P}(x, y)$. For every combination of joint positions the workspace of the manipulator can be divided into four regions based on the sign of the motion of each joint, as shown in Table 1. Similarly, for every combination of joint positions the workspace at the end-effector can also be divided into four regions based on the signs of the joints torques caused by the application of the user force, as shown in Fig. 2b and Table 2.

Table 1 Joint motion of a 2 DOF revolute joint manipulator

\begin{tabular}{lll}
\hline Region & Motion of joint 1 & Motion of joint 2 \\
\hline (1) & + & + \\
(2) & + & - \\
(3) & - & - \\
(4) & - & + \\
\hline
\end{tabular}

Table 2 Joint torques caused by applied user input force

\begin{tabular}{lll}
\hline Region & Torque at joint 1 & Torque at joint 2 \\
\hline $\mathbf{1}$ & + & + \\
$\mathbf{2}$ & + & - \\
$\mathbf{3}$ & - & - \\
$\mathbf{4}$ & - & + \\
\hline
\end{tabular}

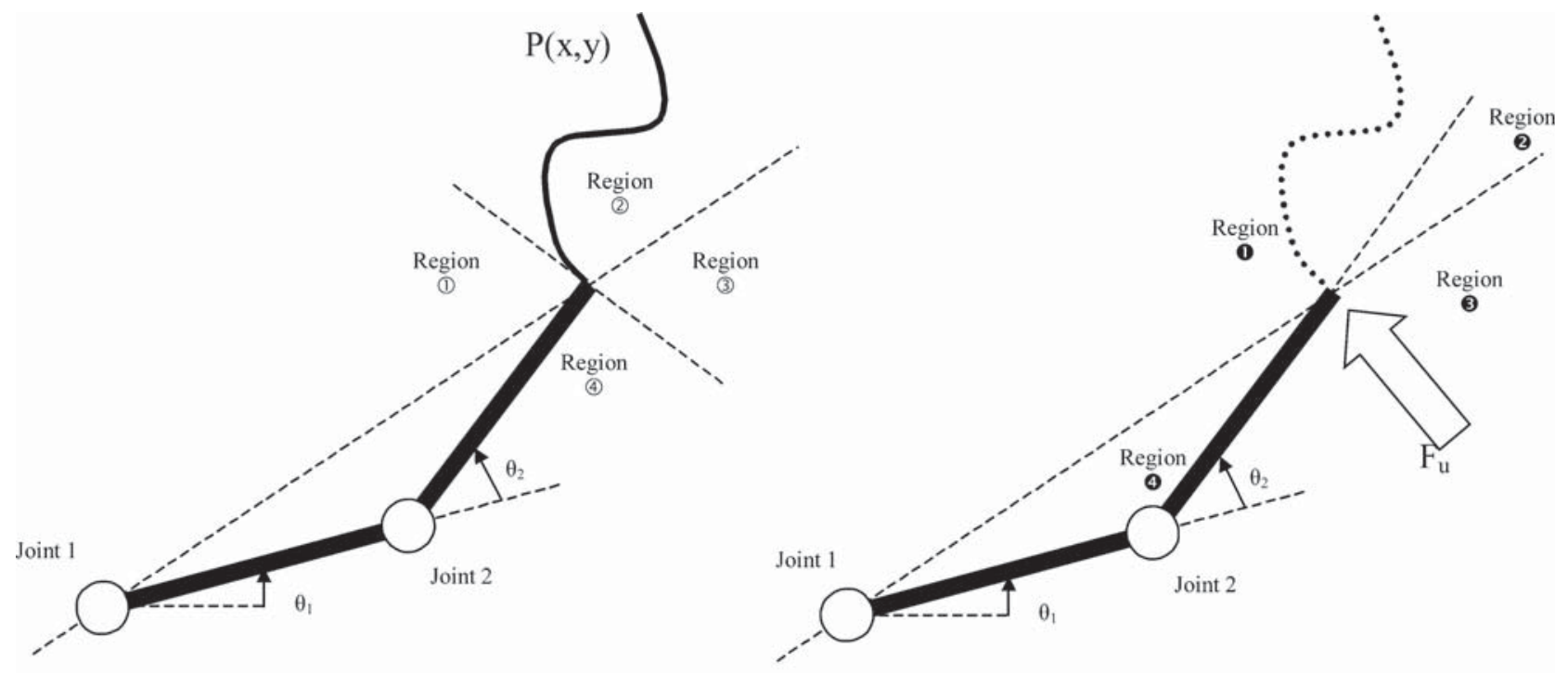

(a)

(b)

Fig. 2 Joint motion of a 2 DOF revolute joint manipulator 
To ensure that the passive robot can move along a desired path it is necessary that the sign of the motion of the joint and the sign of the torque caused by the applied user force are in the same direction. It can be seen from Tables 1 and 2 that motion is possible in region (1), which requires positive directions of $\theta_{1}$ and $\theta_{2}$, only if the user force is directed into region $\mathbf{0}$; i.e. only if the user force produces positive torques at joints 1 and 2 . Similarly, motion is possible into region (2) only when the user force is in region 2 , etc.

It can clearly be seen that if the user tries to apply a force along the desired path, which is intuitive, then no motion of the end-effector may result. For example, for the kinematic configuration shown in Fig. 2b, if the path of the end-effector has a direction within region (2) and the direction of the user force is in region $\mathbf{1}$ along the path, the torques produced at the joints would result in a different direction of the end-effector. Therefore, no motion is allowed by the control system until the direction of the user force is changed to be into region $\mathbf{2}$, which is much smaller than region $\mathbf{P}$ in this particular configuration. Hence, if the user is shown the desired path on a computer screen, which is the case with PADyC, then the initial action of the user is to push in the shown direction of the path and then 'feel' his or her way, by changing the direction of the force, until motion is allowed by the control system. For this, the user must have significant experience to operate the robot effectively.

Subsequently, in this work a control handle that incorporates a pointer mechanism has been implemented. It is used in combination with the control strategy described below to inform the user about the direction

\section{CONTROL STRATEGY}

The aim of the control algorithm is to make the manipulator end-effector track a desired position command when the user-input force is in a specified direction (as described above) while cancelling backlash at the worm interface. In order to ensure operator safety the robot and control method must not exhibit:

(a) any motion against the user;

(b) any motion without direct control from the user;

(c) any backlash at either of the worm/wheel interfaces.

In order to ensure that the robot does not exhibit motion against the user, the robot should only be allowed to move if the torque acting on each joint is in the same direction as the desired motion of that joint. These conditions can be expressed mathematically by equation (1), and for safety reasons they must both be satisfied in order for the robot to be able to move. To implement this strategy successfully, strain gauges have been mounted on each link in order to measure the link torques, $\tau_{\text {act } 1}$ and $\tau_{\text {act2 }}$ :

$$
\operatorname{sgn}\left(\dot{\theta}_{1 \mathrm{~d}}\right)=\operatorname{sgn}\left(\tau_{\mathrm{act} 1}\right) \quad \text { and } \operatorname{sgn}\left(\dot{\theta}_{2 \mathrm{~d}}\right)=\operatorname{sgn}\left(\tau_{\mathrm{act} 2}\right)
$$

As with similar devices, PADyC and PTER, the user controls motion of the end-effector along the path by grasping the control handle and applying a force. Only if the conditions of equation (1) are satisfied is the robot end-effector allowed to move along the path. The desired velocity of the end-effector, $V_{\mathrm{d}}$, is in a direction determined by the path at a magnitude derived from the magnitude of the user input force and the relationship given by

$$
\left|V_{\mathrm{d}}\right|= \begin{cases}0 & \forall\left|\mathbf{F}_{\mathrm{u}}\right|<F_{\mathrm{u}, \text { min }} \\ V_{\mathrm{d}, \max }\left[-\frac{2\left(\left|\mathbf{F}_{\mathrm{u}}\right|-F_{\mathrm{u}, \min }\right)^{3}}{\left(F_{\mathrm{u}, \max }-F_{\mathrm{u}, \min }\right)^{3}}+\frac{3\left(\left|\mathbf{F}_{\mathrm{u}}\right|-F_{\mathrm{u}, \text { min }}\right)^{2}}{\left(F_{\mathrm{u}, \max }-F_{\mathrm{u}, \min }\right)^{2}}\right] & \forall F_{\mathrm{u}, \min } \leqslant\left|\mathbf{F}_{\mathrm{u}}\right| \leqslant F_{\mathrm{u}, \text { max }} \\ V_{\mathrm{d}, \max } & \forall\left|\mathbf{F}_{\mathrm{u}}\right|>F_{\mathrm{u}, \max }\end{cases}
$$

where $V_{\mathrm{d}, \max }, F_{\mathrm{u}, \min }$ and $F_{\mathrm{u} \text {,max }}$ are all positive constants. This velocity-limiting algorithm is also shown graphically in Fig. 4 and the relationship ensures that velocity and acceleration demands for the end-effector are continuous.

In order to cancel backlash in the worm mechanism the following control strategy is used [4]. For motion of a joint in the clockwise direction (i.e. $\dot{\theta}>0$ ) the motor command voltage for the $\alpha$ worm is set to a constant value and the $\beta$ motor torque, $\tau_{\mathrm{m} \beta n}$, is controlled to unwind the $\beta$ worm to track the trajectory. In this condition the $\beta$ worm leads and the $\alpha$ worm is used to follow the motion of the worm wheel without applying unnecessary frictional forces to the system. Control of motion in this manner shall be termed $\beta$-unwinding control. However, in the anticlockwise direction (i.e. $\dot{\theta}<0$ ) the $\beta$ worm motor command voltage is set to a constant value and $\tau_{\mathrm{m} \alpha n}$ is used to control the unwinding of the $\alpha$ worm to track 


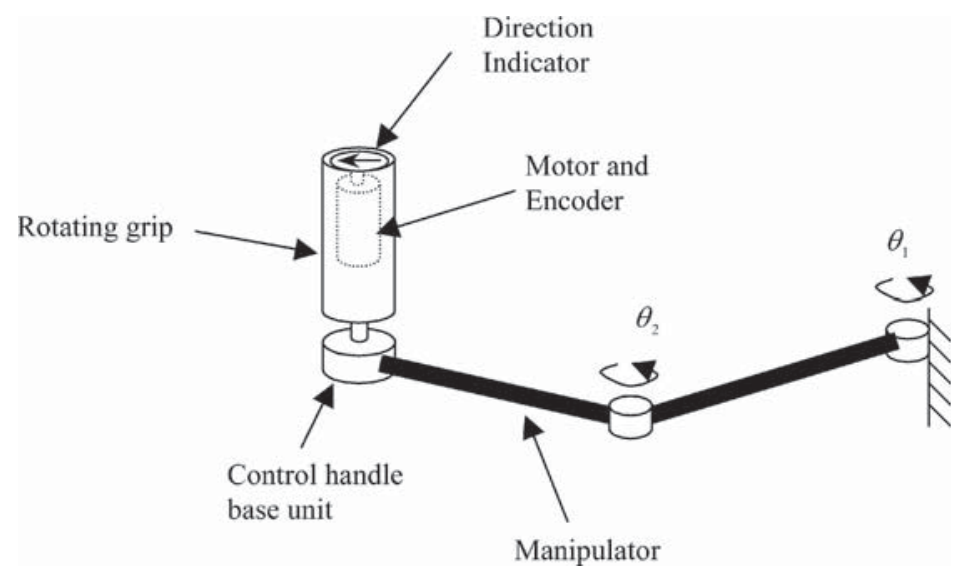

Fig. 3 Manipulator control handle schematic

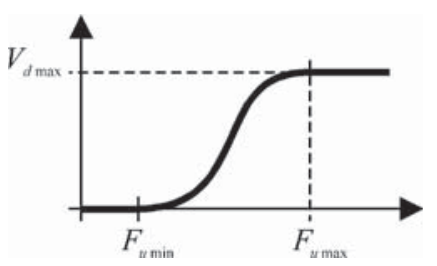

Fig. 4 User-defined velocity demand

the trajectory. This is termed $\alpha$-unwinding control. In this instance, the $\alpha$ worm leads and the $\beta$ worm follows.

\section{MATHEMATICAL MODEL}

Consider the 2 DOF revolute joint, planar manipulator shown in Fig. 5. In general the dynamic equation for the system is given by the following equation, where $\boldsymbol{\tau}$ is a vector of joint torque provided by the joint actuators, $\boldsymbol{F}_{\mathrm{u}}$ is the user-input force and $\mathbf{J}$ is the arm Jacobian matrix for the manipulator:

$$
\mathbf{M} \ddot{\boldsymbol{q}}+\mathbf{C} \dot{\boldsymbol{q}}+\mathbf{V}_{\mathrm{m}} \dot{\boldsymbol{q}}=\boldsymbol{\tau}+\mathbf{J}^{\mathrm{T}} \boldsymbol{F}_{\mathrm{u}}
$$

From the model of the worm-driven joint [4], the torque provided by the mechanisms for joints 1 and 2 can be written respectively

$$
\begin{aligned}
\tau_{1}= & \frac{\tau_{\mathrm{m} \alpha 1} r_{\theta 1}}{r_{\alpha 1} \Delta_{\alpha 1}}+\frac{\tau_{\mathrm{m} \beta 1} r_{\theta 1}}{r_{\beta 1} \Delta_{\beta 1}}-J_{\alpha 1} \frac{\ddot{\theta}_{1} r_{\theta 1}^{2}}{\Delta_{\alpha 1} r_{\alpha 1}^{2} \tan \gamma_{1}} \\
& -J_{\beta 1} \frac{\ddot{\theta}_{1} r_{\theta 1}^{2}}{\Delta_{\beta 1} r_{\beta 1}^{2} \tan \gamma_{1}}-C_{\mathrm{f} \alpha 1} \frac{\dot{\theta}_{1} r_{\theta 1}^{2}}{\Delta_{\alpha 1} r_{\alpha 1}^{2} \tan \gamma_{1}} \\
& -C_{\mathrm{f} \beta 1} \frac{\dot{\theta}_{1} r_{\theta 1}^{2}}{\Delta_{\beta 1} r_{\beta 1}^{2} \tan \gamma_{1}} \\
\tau_{2}= & \frac{\tau_{\mathrm{m} \alpha 2} r_{\theta 2}}{r_{\alpha 2} \Delta_{\alpha 2}}+\frac{\tau_{\mathrm{m} \beta 2} r_{\theta 2}}{r_{\beta 2} \Delta_{\beta 2}}-J_{\alpha 2} \frac{\ddot{\theta}_{2} r_{\theta 2}^{2}}{\Delta_{\alpha 2} r_{\alpha 2}^{2} \tan \gamma_{2}} \\
& -J_{\beta 2} \frac{\ddot{\theta}_{2} r_{\theta 2}^{2}}{\Delta_{\beta 2} r_{\beta 2}^{2} \tan \gamma_{2}}-C_{\mathrm{f} \alpha 2} \frac{\dot{\theta}_{2} r_{\theta 2}^{2}}{\Delta_{\alpha 2} r_{\alpha 2}^{2} \tan \gamma_{2}} \\
& -C_{\mathrm{f} \beta 2} \frac{\dot{\theta}_{2} r_{\theta 2}^{2}}{\Delta_{\beta 2} r_{\beta 2}^{2} \tan \gamma_{2}}
\end{aligned}
$$

The terms $\Delta_{\alpha n}$ and $\Delta_{\beta n}$ in equations (4) and (5) represent the torque transfer ratio between the worms and the worm

where

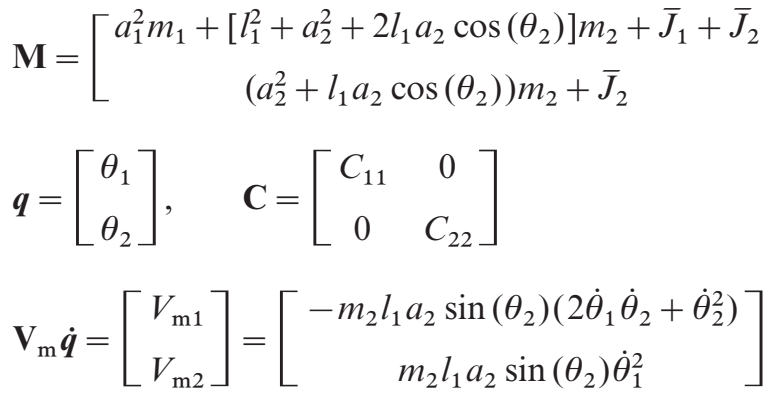

$$
\begin{aligned}
& \mathbf{J}=\left[\begin{array}{cc}
-l_{1} \sin \left(\theta_{1}\right)-l_{2} \sin \left(\theta_{1}+\theta_{2}\right) & -l_{2} \sin \left(\theta_{1}+\theta_{2}\right) \\
l_{1} \cos \left(\theta_{1}\right)+l_{2} \cos \left(\theta_{1}+\theta_{2}\right) & l_{2} \cos \left(\theta_{1}+\theta_{2}\right)
\end{array}\right] \\
& \boldsymbol{\tau}=\left[\begin{array}{c}
\tau_{1} \\
\tau_{2}
\end{array}\right] \quad \text { and } \quad \boldsymbol{F}_{\mathrm{n}}=\left[\begin{array}{c}
F_{\mathrm{n} x} \\
F_{\mathrm{u} y}
\end{array}\right]
\end{aligned}
$$

wheel of joint $n$. These terms include an exponential stick-slip model of friction in the dual worm mechanism of joint $n$, as shown by

$$
\begin{aligned}
& \Delta_{\alpha n}=\frac{\sin \gamma_{n}+\left[\mu_{\mathrm{d} \alpha n}+\left(\mu_{\mathrm{s} \alpha n}-\mu_{\mathrm{d} \alpha n}\right) \mathrm{e}^{\mid \dot{\theta}_{n} / /\left(\varepsilon_{n} \sin \gamma_{n}\right)}\right] \operatorname{sgn}\left(\dot{\theta}_{n}\right) \cos \gamma_{n}}{\cos \gamma_{n}-\left[\mu_{\mathrm{d} \alpha n}+\left(\mu_{\mathrm{s} \alpha n}-\mu_{\mathrm{d} \alpha n}\right) \mathrm{e}^{\mid \dot{\theta}_{n} / /\left(\varepsilon_{n} \sin \gamma_{n}\right)}\right] \operatorname{sgn}\left(\dot{\theta}_{n}\right) \sin \gamma_{n}} \\
& \Delta_{\beta n}=\frac{\sin \gamma_{n}-\left[\mu_{\mathrm{d} \beta n}+\left(\mu_{\mathrm{s} \beta n}-\mu_{\mathrm{d} \beta n}\right) \mathrm{e}^{\left|\dot{\theta}_{n}\right| /\left(\varepsilon_{n} \sin \gamma_{n}\right)}\right] \operatorname{sgn}\left(\dot{\theta}_{n}\right) \cos \gamma_{n}}{\cos \gamma_{n}+\left[\mu_{\mathrm{d} \beta n}+\left(\mu_{\mathrm{s} \beta n}-\mu_{\mathrm{d} \beta n}\right) \mathrm{e}^{\left|\dot{\theta}_{n}\right| /\left(\varepsilon_{n} \sin \gamma_{n}\right)}\right] \operatorname{sgn}\left(\dot{\theta}_{n}\right) \sin \gamma_{n}}
\end{aligned}
$$




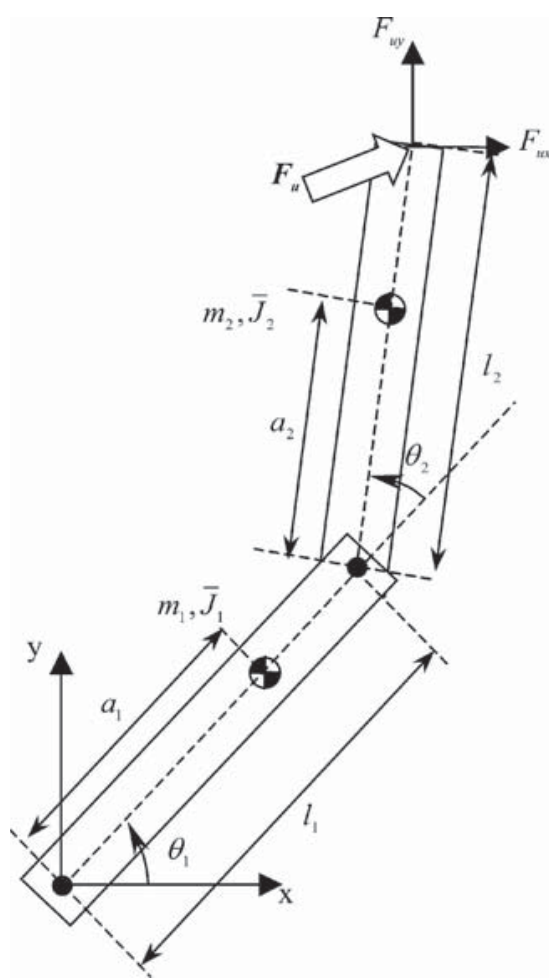

Fig. 5 A two DOF planar manipulator

Substitution of the joint torque equations (4) and (5) into the system equation of motion (3) yields

$$
\mathbf{M} \ddot{\boldsymbol{q}}+\mathbf{M}_{\mathrm{r}} \ddot{\boldsymbol{q}}+\mathbf{C} \dot{\boldsymbol{q}}+\mathbf{C}_{\mathrm{r}} \dot{\boldsymbol{q}}+\mathbf{V}_{\mathrm{m}} \dot{\boldsymbol{q}}=\mathbf{B} \boldsymbol{\tau}_{\mathrm{m}}+\mathbf{J}^{\mathrm{T}} \boldsymbol{F}_{\mathrm{u}}
$$

where

$$
\begin{aligned}
& \mathbf{M}_{\mathrm{r}}=\left[\begin{array}{c}
\frac{J_{\alpha 1} r_{\theta 1}^{2}}{\Delta_{\alpha 1} r_{\alpha 1}^{2} \tan \gamma_{1}}+\frac{J_{\beta 1} r_{\theta 1}^{2}}{\Delta_{\beta 1} r_{\beta 1}^{2} \tan \gamma_{1}} \\
0
\end{array}\right. \\
& \mathbf{C}_{\mathrm{r}}=\left[\begin{array}{c}
\frac{C_{\mathrm{f} \alpha 1} r_{\theta 1}^{2}}{\Delta_{\alpha 1} r_{\alpha 1}^{2} \tan \gamma_{1}}+\frac{C_{\mathrm{f} \beta 1} r_{\theta 1}^{2}}{\Delta_{\beta 1} r_{\beta 1}^{2} \tan \gamma_{1}} \\
0
\end{array}\right. \\
& \mathbf{B}=\left[\begin{array}{cccc}
\frac{r_{\theta 1}}{\Delta_{\alpha 11} r_{\alpha 1}} & \frac{r_{\theta 1}}{\Delta_{\beta 11} r_{\beta 1}} & 0 & 0 \\
0 & 0 & \frac{r_{\theta 2}}{\Delta_{\alpha 12} r_{\alpha 2}} & \frac{r_{\theta 2}}{\Delta_{\beta 12} r_{\beta 2}}
\end{array}\right] \\
& =\left[\begin{array}{cccc}
B_{11} & B_{12} & 0 & 0 \\
0 & 0 & B_{23} & B_{24}
\end{array}\right] \\
& \boldsymbol{\tau}_{\mathrm{m}}=\left[\tau_{\mathrm{m} \alpha 1} \tau_{\mathrm{m} \beta 1} \tau_{\mathrm{m} \alpha 2} \tau_{\mathrm{m} \beta 2}\right]^{\mathrm{T}}
\end{aligned}
$$$$
\left.\frac{J_{\alpha 2} r_{\theta 2}^{2}}{\Delta_{\alpha 2} r_{\alpha 2}^{2} \tan \gamma_{2}}+\frac{J_{\beta 2} r_{\theta 2}^{2}}{\Delta_{\beta 2} r_{\beta 2}^{2} \tan \gamma_{2}}\right]
$$$$
\left.\frac{C_{\mathrm{f} \alpha 2} r_{\theta 2}^{2}}{\Delta_{\alpha 2} r_{\alpha 2}^{2} \tan \gamma_{2}}+\frac{C_{\mathrm{f} \beta 2} r_{\theta 2}^{2}}{\Delta_{\beta 2} r_{\beta 2}^{2} \tan \gamma_{2}}\right]=\left[\begin{array}{cc}
\mathbf{C}_{\mathrm{r} 11} & 0 \\
0 & \mathbf{C}_{\mathrm{r} 22}
\end{array}\right]
$$$$
\mathbf{E}=\left[\begin{array}{c}
e_{1} \\
e_{2}
\end{array}\right]=\boldsymbol{q}_{\mathrm{d}}-\boldsymbol{q}
$$

\section{COMPUTED-TORQUE CONTROLLER}

The difference between the desired trajectory and the actual trajectory is defined as

in this manner mathematically decouples the dynamic equations because the forces acting on each joint are explicitly measured. In particular, the forces caused by Coriolis efrect are measured. However, ment of the totas in includes the inertia of the worm wheel, the main shaft and a small portion of the link between the joint and the strain gauge module. Therefore, the torque vector measured by the two link strain gauge sensors is given as

$$
\boldsymbol{\tau}_{\text {act }}=-\left(\mathbf{M}-\mathbf{M}_{\theta}\right) \ddot{\boldsymbol{q}}-\mathbf{V}_{\mathrm{m}} \dot{\boldsymbol{q}}+\mathbf{J}^{\mathrm{T}} \boldsymbol{F}_{\mathrm{u}}
$$

Equation (9) can also be written as

$$
\mathbf{J}^{\mathrm{T}} \boldsymbol{F}_{\mathrm{u}}=\boldsymbol{\tau}_{\mathrm{act}}+\left(\mathbf{M}-\mathbf{M}_{\theta}\right) \ddot{\boldsymbol{q}}+\mathbf{V}_{\mathrm{m}} \ddot{\boldsymbol{q}}
$$

where

$$
\mathbf{M}_{\theta}=\left[\begin{array}{cc}
J_{\theta 1} & 0 \\
0 & J_{\theta 2}
\end{array}\right], \quad \boldsymbol{\tau}_{\text {act }}=\left[\tau_{\text {act } 1} \tau_{\text {act } 2}\right]^{\mathrm{T}}
$$

and $J_{\theta n}$ represents the residual inertia of link $n$. Combining equations (8) and (10) yields the decoupled system namics given by

$$
\left(\mathbf{M}_{\theta}+\mathbf{M}_{\mathrm{r}}\right) \ddot{\boldsymbol{q}}+\left(\mathbf{C}+\mathbf{C}_{\mathrm{r}}\right) \dot{\boldsymbol{q}}=\mathbf{B} \boldsymbol{\tau}_{\mathrm{m}}+\boldsymbol{\tau}_{\text {act }}
$$

However, due to the placement of the strain gauges on each link, it is possible to simplify equation (8) by introducing the terms $\tau_{\text {act1 }}$ and $\tau_{\text {act2 }}$ which represent measurements from the two sensors. The use of the sensors 
trajectory it is possible to select the computed-torque control law as

$$
\begin{gathered}
\tau_{\mathrm{m} \alpha 1}=\frac{1}{B_{11}}\left[\left(J_{\theta 1}+\frac{J_{\alpha 1} r_{\theta 1}^{2}}{\Delta_{\alpha 1} r_{\alpha 1}^{2} \tan \gamma_{1}}+\frac{J_{\beta 1} r_{\theta 1}^{2}}{\Delta_{\beta 1} r_{\beta 1}^{2} \tan \gamma_{1}}\right)\left(\ddot{\theta}_{\mathrm{d} 1}+u_{\alpha 1}\right)\right. \\
\left.+\left(C_{11}+C_{\mathrm{r} 11}\right) \dot{\theta}_{1}-B_{12} \tau_{\mathrm{m} \beta 1}-\tau_{\mathrm{act} 1}\right]
\end{gathered}
$$

where $u_{\alpha 1}$ is the proportional + integral + derivative (PID) control law given by

$$
u_{\alpha 1}=K_{\mathrm{p} \alpha 1} e_{1}+K_{\mathrm{v} \alpha 1} \dot{e}_{1}+K_{\mathrm{i} \alpha 1} \int e_{1} \mathrm{~d} t
$$

\subsection{Joint $1 \beta$-unwinding controller}

Similarly, $\tau_{\mathrm{m} \beta 1}$ is used to regulate position for $\beta$-unwinding control of joint 1 . Hence the computed-torque PID controller is given by

$$
\begin{aligned}
\tau_{\mathrm{m} \beta 1}=\frac{1}{B_{12}}[ & \left(J_{\theta 1}+\frac{J_{\alpha 1} r_{\theta 1}^{2}}{\Delta_{\alpha 1} r_{\alpha 1}^{2} \tan \gamma_{1}}+\frac{J_{\beta 1} r_{\theta 1}^{2}}{\Delta_{\beta 1} r_{\beta 1}^{2} \tan \gamma_{1}}\right)\left(\ddot{\theta}_{\mathrm{d} 1}+u_{\beta 1}\right) \\
& \left.+\left(C_{11}+C_{\mathrm{r} 11}\right) \dot{\theta}_{1}-B_{11} \tau_{\mathrm{m} \alpha 1}-\tau_{\mathrm{act} 1}\right]
\end{aligned}
$$

where $u_{\beta 1}$ is the PID control law given by

$$
u_{\beta 1}=K_{\mathrm{p} \beta 1} e_{1}+K_{\mathrm{v} \beta 1} \dot{e}_{1}+K_{\mathrm{i} \beta 1} \int e_{1} \mathrm{~d} t
$$

\subsection{Joint $2 \alpha$-unwinding controller}

For $\alpha$-unwinding control of joint 2, the computedtorque PID controller is chosen in a similar manner and is given by

$$
\begin{gathered}
\tau_{\mathrm{m} \alpha 2}=\frac{1}{B_{23}}\left[\left(J_{\theta 2}+\frac{J_{\alpha 2} r_{\theta 2}^{2}}{\Delta_{\alpha 2} r_{\alpha 2}^{2} \tan \gamma_{2}}+\frac{J_{\beta 2} r_{\theta 2}^{2}}{\Delta_{\beta 2} r_{\beta 2}^{2} \tan \gamma_{2}}\right)\left(\ddot{\theta}_{\mathrm{d} 2}+u_{\alpha 2}\right)\right. \\
\left.+\left(C_{22}+C_{\mathrm{r} 22}\right) \dot{\theta}_{2}-B_{24} \tau_{\mathrm{m} \beta 1}-\tau_{\mathrm{act} 2}\right]
\end{gathered}
$$

where $u_{\alpha 2}$ is the PID control law given by

$$
u_{\alpha 2}=K_{\mathrm{p} \alpha 2} e_{2}+K_{\mathrm{v} \alpha 2} \dot{e}_{2}+K_{\mathrm{i} \alpha 2} \int e_{2} \mathrm{~d} t
$$

\subsection{Joint $2 \beta$-unwinding controller}

The $\beta$-unwinding control of joint 2 is accomplished using the computed-torque PID controller of the following equations:

$$
\begin{gathered}
\tau_{\mathrm{m} \beta 2}=\frac{1}{B_{24}}\left[\left(J_{\theta 2}+\frac{J_{\alpha 2} r_{\theta 2}^{2}}{\Delta_{\alpha 2} r_{\alpha 2}^{2} \tan \gamma_{2}}+\frac{J_{\beta 2} r_{\theta 2}^{2}}{\Delta_{\beta 2} r_{\beta 2}^{2} \tan \gamma_{2}}\right)\left(\ddot{\theta}_{\mathrm{d} 2}+u_{\beta 2}\right)\right. \\
\left.+\left(C_{22}+C_{\mathrm{r} 22}\right) \dot{\theta}_{2}-B_{23} \tau_{\mathrm{m} \alpha 1}-\tau_{\mathrm{act} 2}\right]
\end{gathered}
$$

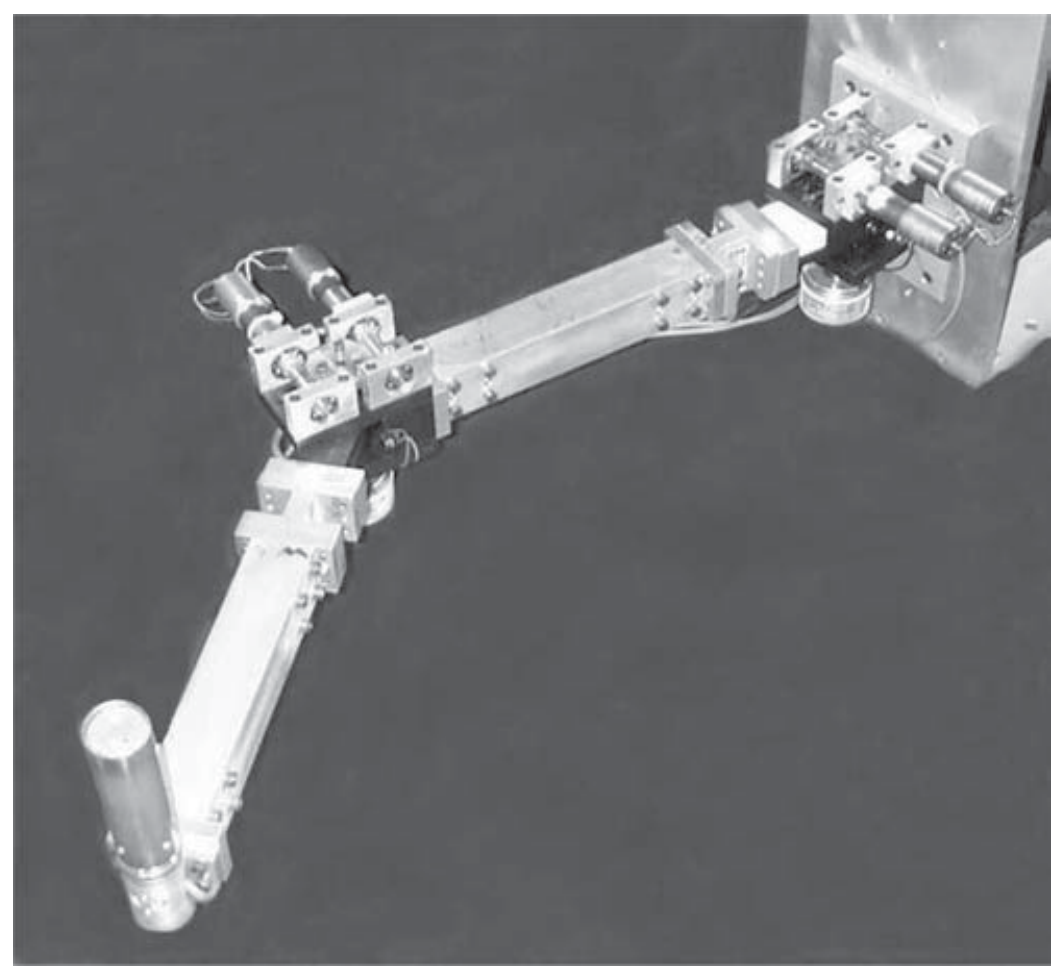

Fig. 6 A two DOF manipulator 


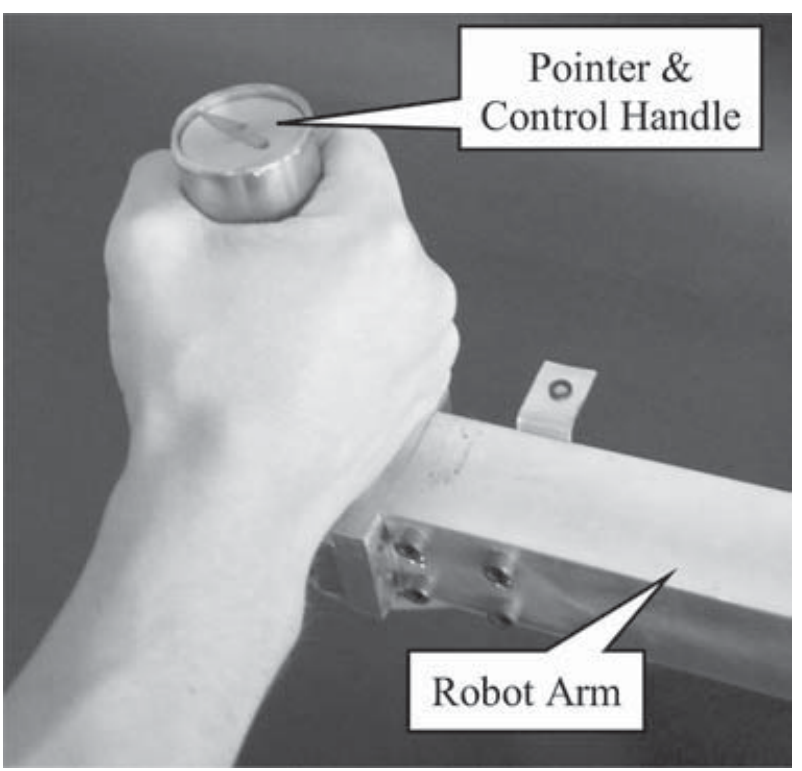

Fig. 7 Control handle and pointer mechanism where $u_{\beta 2}$ is the PID control law given by

$$
u_{\beta 2}=K_{\mathrm{p} \beta 2} e_{2}+K_{\mathrm{v} \beta 2} \dot{e}_{2}+K_{\mathrm{i} \beta 2} \int e_{2} \mathrm{~d} t
$$

\section{EXPERIMENTAL SET-UP}

The 2 DOF dual-worm driven joint planar manipulator is shown in Fig. 6. The control handle and pointer mechanism are shown in more detail in Fig. 7. Control of the robot is performed using a Pentium $233 \mathrm{MHz}$ personal computer running the QNX 4.25 real-time operating system. A schematic diagram of the control system is shown in Fig. 8.

Each control algorithm requires measurements of motor torque, joint torque and joint position. The torque acting on each joint is measured using four strain gauges, in a Wheatstone bridge configuration, mounted on a specially designed section of the link. Each joint uses a pair of

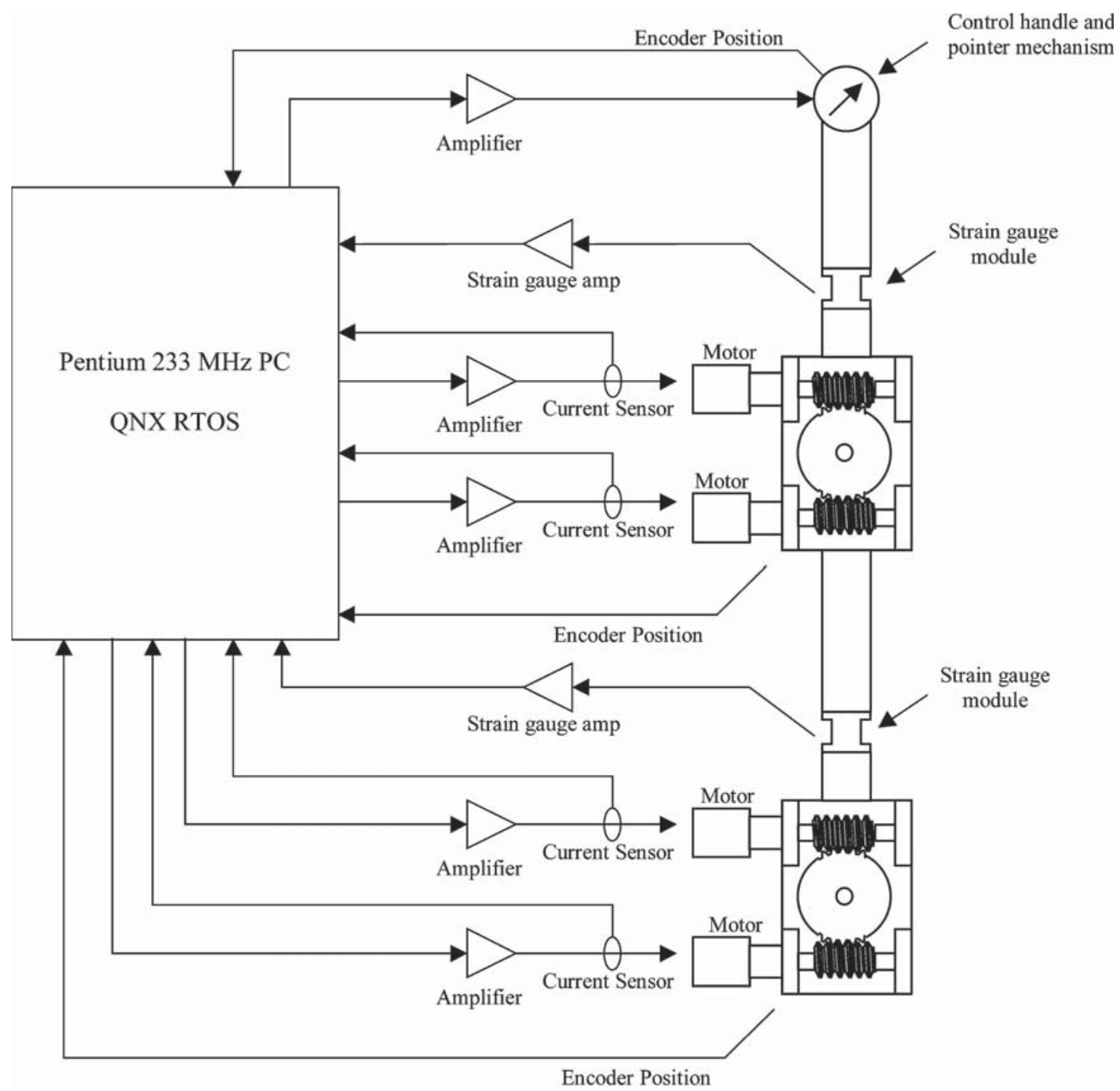

Fig. 8 Shematic diagram of the control system 


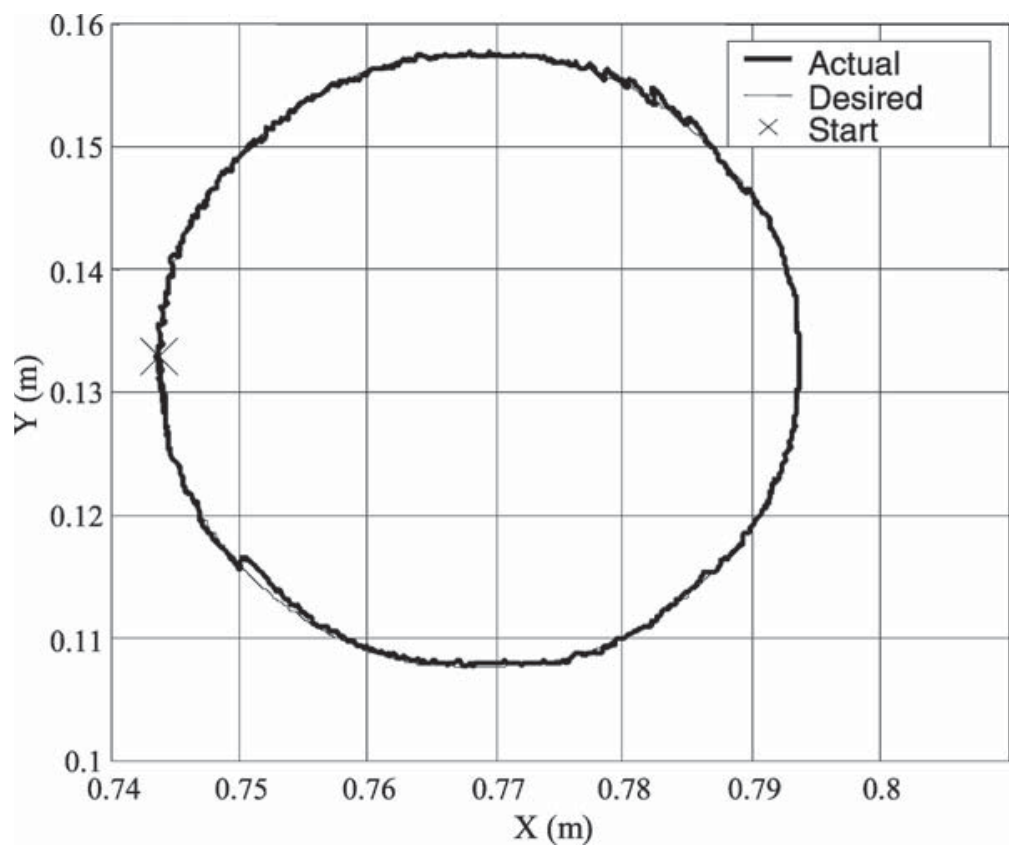

Fig. 9 Tracking of a circular path

a) Time history of X-position

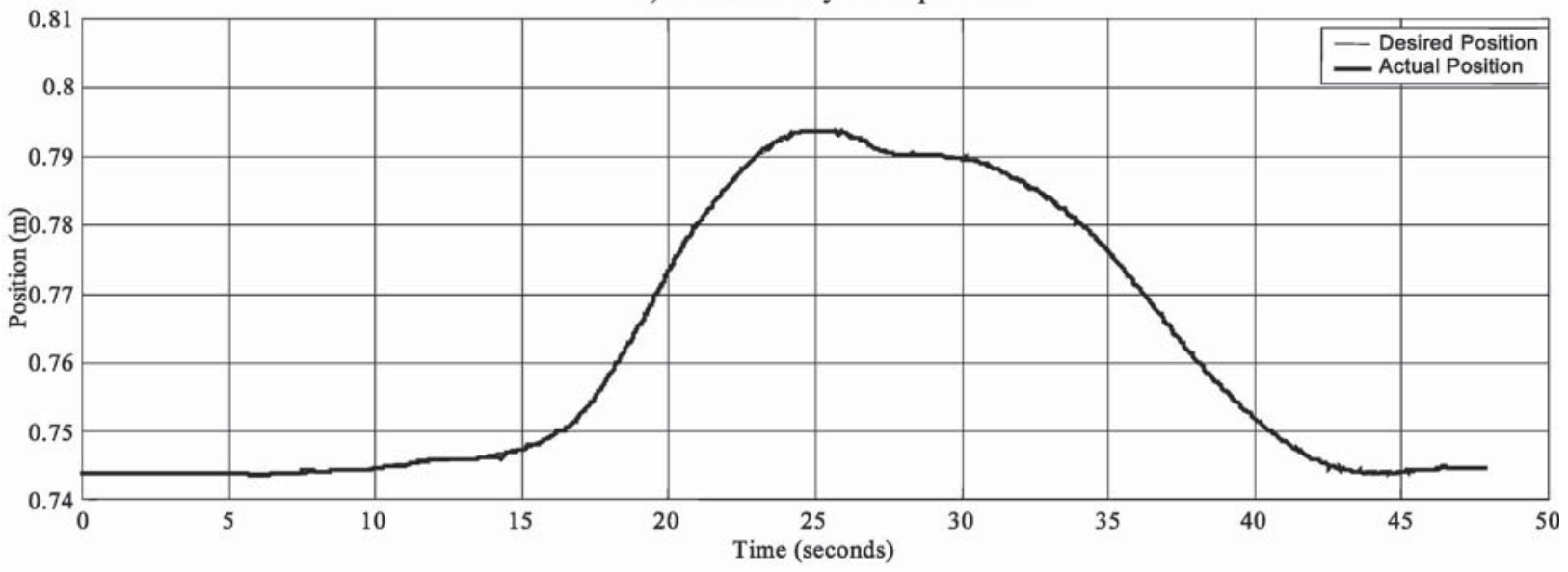

b) Time history of Y-position

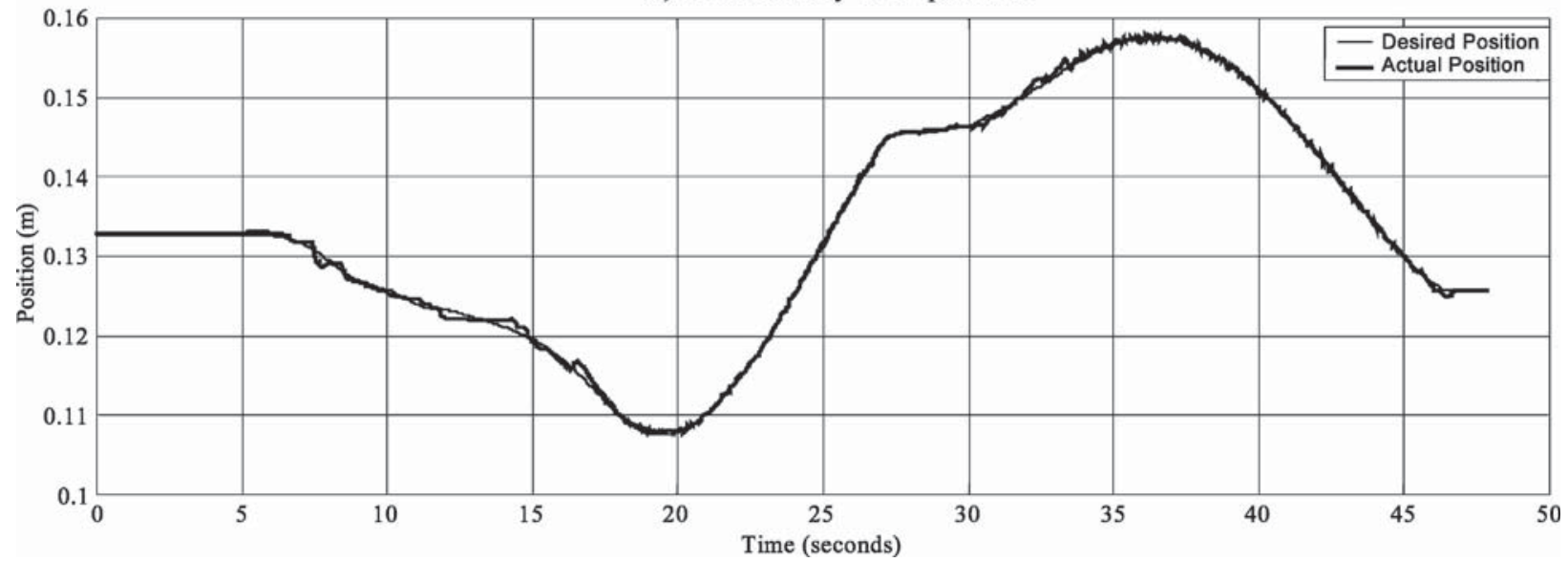

Fig. 10 Time history of the end-effector $X$ and $Y$ positions for the circular path shown in Fig. 9 
geared d.c. motors selected to give a peak joint speed of only $2 \mathrm{r} / \mathrm{min}$. Closed-loop regulation of the motor output torque is accomplished by measuring the armature current and implementing a digital PI (proportional + integral) controller. All analogue signals are measured and generated using 12-bit analogue-to-digital converters and 12-bit digital-to-analogue converters. The link rotation is recorded using an encoder and appropriate electronics to generate 20000 counts per revolution, giving a resolution of $3.142 \times 10^{-4} \mathrm{rads} /$ count (or $0.018^{\circ}$ count) for each joint. The link rotational velocity measurement is obtained in software by using a backwarddifference algorithm.

\section{RESULTS AND DISCUSSION}

To test the ability of the manipulator end-effector to track a trajectory, a circular path of diameter $50 \mathrm{~mm}$ was chosen. The parameters used to compensate for system dynamics in the control algorithm are given in Table 3. It should be noted that the static and dynamic friction coefficients at the two joints are different because they are dependent on the physical condition of the joint mechanism and gear interface, including the surface condition and lubrication. They were obtained experimentally.

The desired and actual paths of the end-effector, expressed in Cartesian coordinates $x$ and $y$, are shown in Fig. 9. The start position of the end-effector on the path is also illustrated. Figure 10 shows the circular path expressed as the time history of both the $x$ and $y$ positions of the end-effector. It can be seen that the end-effector of the robot tracks the path with little error. While the plot of the $x$ position illustrates good tracking performance it can be seen that the graph of the $y$ position shows some deviation from the path. Figure 11 illustrates the error in the position of the end-effector shown in Cartesian coordinates. The $x$-axis error, shown in Fig. 11a, does not exceed $0.6 \mathrm{~mm}$ at any time while the $y$-axis error (Fig. 11b) remains under $1.5 \mathrm{~mm}$. Figure 12 shows the components of the force calculated from the joint torque recorded by the strain gauges on each link.

The difference in the magnitudes of the error in the $x$ and $y$ positions depends on the particular configuration of the manipulator with respect to the chosen directions of
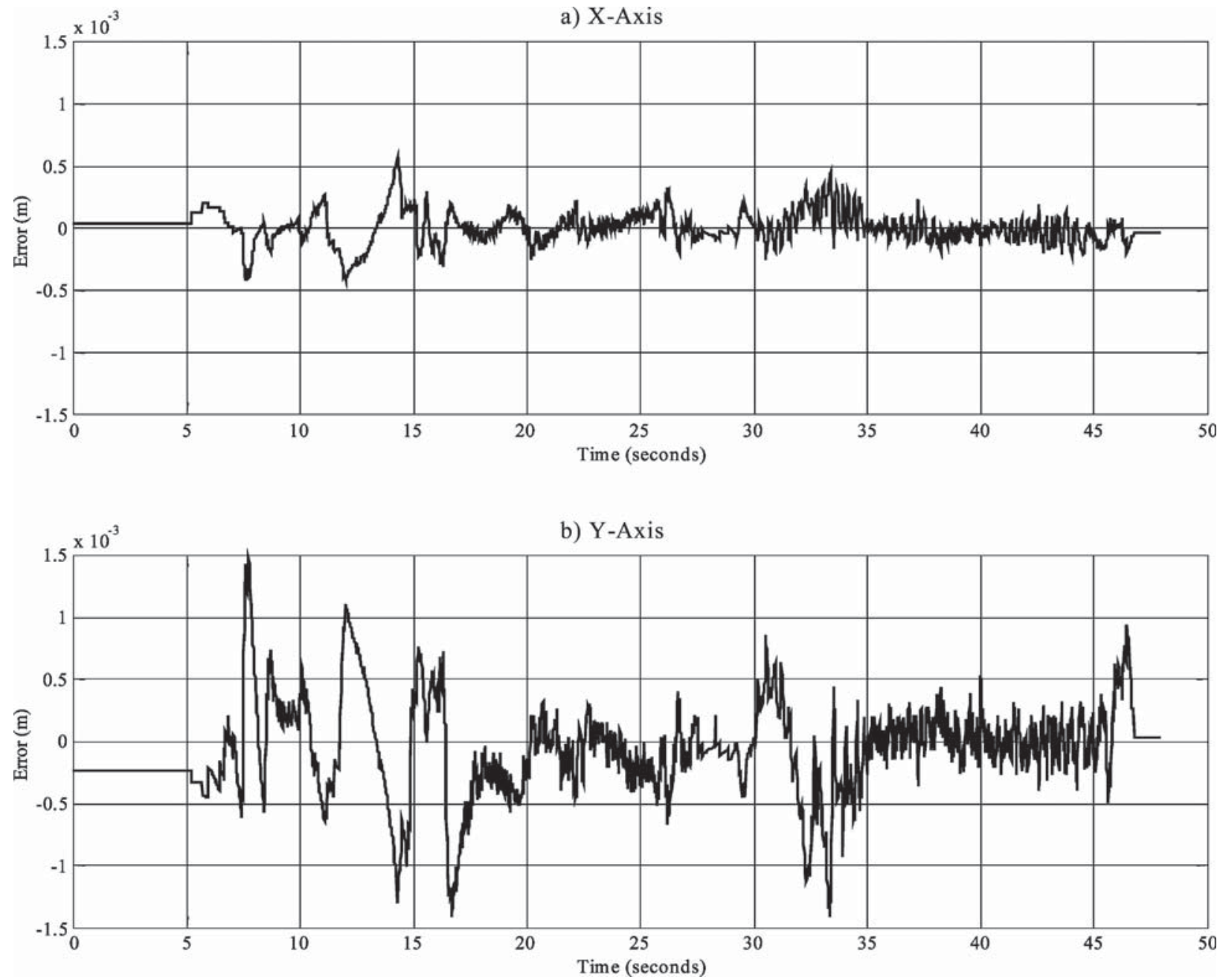

Fig. 11 End-effector position error 
Table 3 System parameters

\begin{tabular}{llll}
\hline & Worm wheel & $\alpha$ worm & $\beta$ worm \\
\hline Inertia $\left(\mathrm{kg} \mathrm{m}^{2}\right)$ & $J_{\theta 1}=J_{\theta 2}=0.25122$ & $J_{\alpha 1}=J_{\alpha 2}=0.003698$ & $J_{\beta 1}=J_{\beta 2}=0.003698$ \\
Viscous damping (N m s/rad) & $C_{\mathrm{f} \theta 1}=C_{\mathrm{f} \theta 2}=0.005$ & $C_{\mathrm{f} \alpha 1}=C_{\mathrm{f} \alpha 2}=0.001$ & $C_{\mathrm{f} \beta 1}=C_{\mathrm{f} \beta 2}=0.001$ \\
Gear pitch circle diameter (PCD) $(\mathrm{m})$ & $r_{\theta 1}=r_{\theta 2}=0.025$ & $r_{\alpha 1}=r_{\alpha 2}=0.0065$ & $r_{\beta 1}=r_{\beta 2}=0.0065$ \\
Static friction & & $\mu_{\mathrm{s} \alpha 1}=\mu_{\mathrm{s} \alpha 2}=0.12$ & $\mu_{\mathrm{s} \beta 1}=\mu_{\mathrm{s} \beta 2}=0.16$ \\
Dynamic friction & & $\mu_{\mathrm{d} \alpha 1}=\mu_{\mathrm{d} \alpha 2}=0.10$ & $\mu_{\mathrm{d} \beta 1}=\mu_{\mathrm{d} \beta 2}=0.14$ \\
Friction constant & & $\varepsilon_{1}=\varepsilon_{2}=0.001$ & $\varepsilon_{1}=\varepsilon_{2}=0.001$ \\
Worm lead angle & & $\gamma_{1}=\gamma_{2}=0.05236$ & $\gamma_{1}=\gamma_{2}=0.05236$ \\
Joint link lengths (m) & & & \\
& $l_{1}=0.450$ & & \\
\hline
\end{tabular}

a) X-Axis

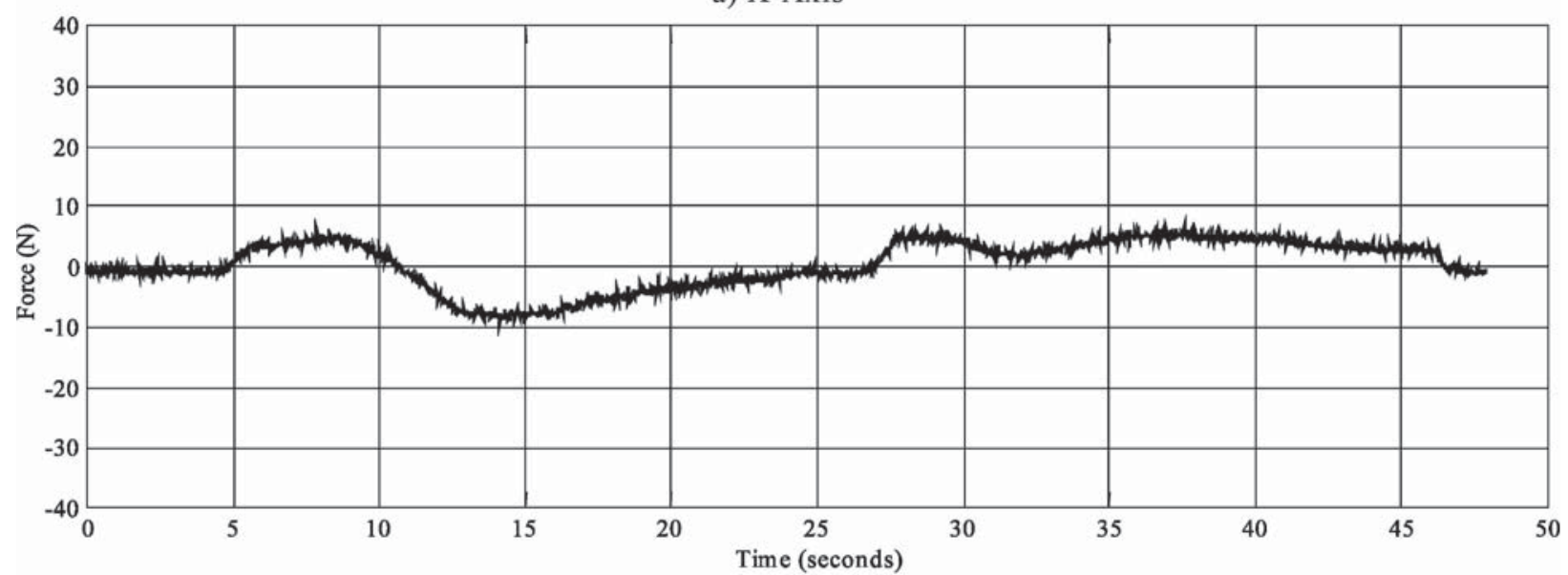

b) Y-Axis

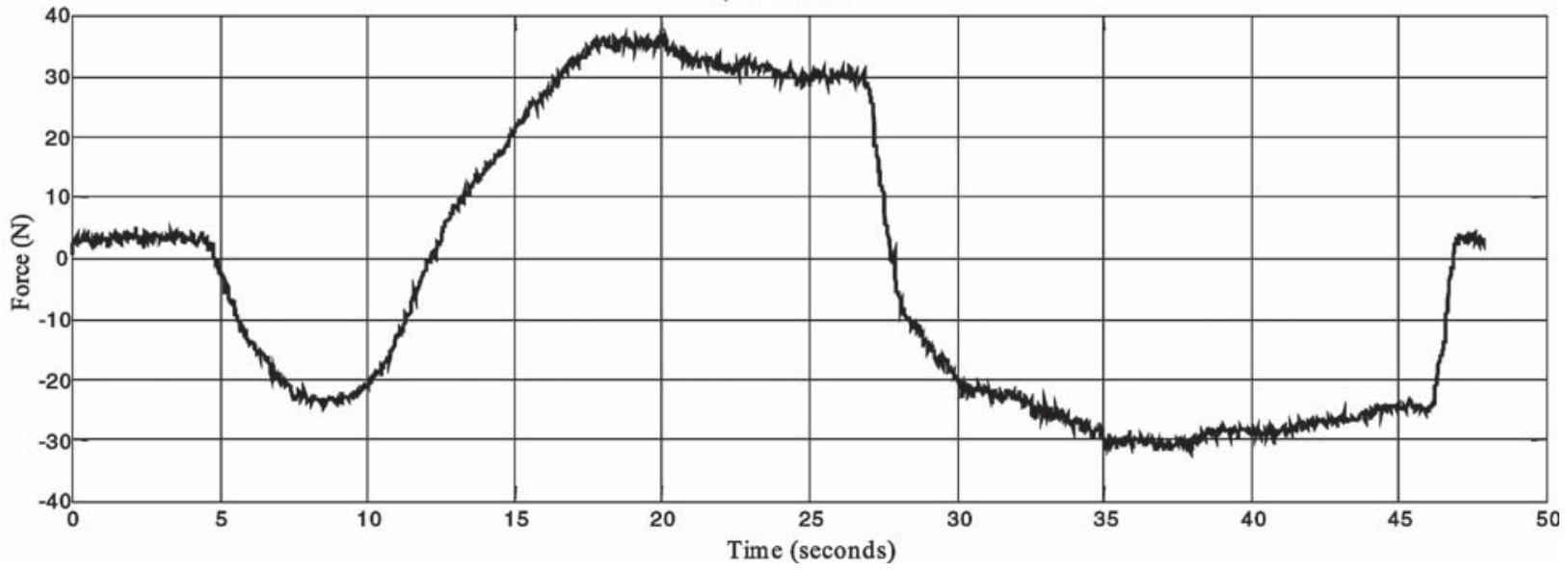

Fig. 12 End-effector force computed from the joint torques generated when the path shown in Fig. 9 is followed

the $x$ and $y$ axes, and is attributed to the flexibility in the mechanism of joint 1 . The end-effector error, caused by system compliance, is dependent upon the manipulator configuration but can be highlighted in a static test. For example, the test for a particular configuration has shown that for every $10 \mathrm{~N}$ of user-input force in the $y$ direction the end-effector is deflected by approximately $1.15 \mathrm{~mm}$ in the $y$ axis in the given manipulator configuration. For the same configuration and force in the $x$ direction the end-effector is deflected by only $0.1 \mathrm{~mm}$ in the $x$ axis. By increasing the stiffness of the main shaft of joint 1 the error in the $y$ axis due to flexibility can be significantly reduced.

Using these control strategies the tracking error over the $50 \mathrm{~mm}$ diameter circular path was shown to be less than $1.6 \mathrm{~mm}$ in magnitude and was caused by the interaction of the unmodelled joint flexibility, user applied force and the controller. In view of the large errors that could be obtained due to the application of a force by the user (approximately $4 \mathrm{~mm}$ for $35 \mathrm{~N}$ ), this is considered to be reasonable. However, since the user can easily apply forces to the control handle in the order of

Proc. Instn Mech. Engrs Vol. 218 Part I: J. Systems and Control Engineering 
$50 \mathrm{~N}$, the error shown in these experiments with this 2 DOF manipulator cannot be tolerated in most medical applications without reducing the effect of the stiffness and/or introducing instrumentation to raise an alarm whenever the user exceeds a maximum allowable force.

\section{CONCLUSIONS}

It has been shown that by implementing a pointing device to direct the user to apply a force in the correct way the end-effector of a 2 DOF manipulator can be guided along a predefined path under the direct physical control of a human operator. PADyC uses a computer monitor to display the desired path and requires the user to have some knowledge/experience of the system and its behaviour in order to successfully negotiate the corridors that describe the path [1]. A distinct advantage of using the pointing device presented in this work is that the user is shown how to apply a force to the control handle in order to accomplish the desired task more efficiently. Therefore it is not necessary for the user to make the decision about how to apply the force based on learned knowledge of the system, significantly reducing the need for training. However, control of the $2 \mathrm{DOF}$ manipulator with the strategies described in this paper requires measurement of the user-input force and implementation of a pointing device. The increase in tracking performance over other solutions far outweighs the increased cost incurred by the addition of force measurement and pointing devices.
Safe deployment of the end-effector has been assured by allowing motion only if the direction of the applied user-input force would allow the controller to restrict motion along the predefined path. The effects of system compliance have been shown to have an adverse affect on controller performance. However, a mechanical redesign of the system and reconfiguration of the manipulator would result in increased robustness to large user-input forces. Using the control strategy described in this paper it would also be possible to replace the motorized joints with brake or clutch mechanisms without loss of performance. Future work will address the implementation of these control strategies on a passive manipulator.

\section{REFERENCES}

1 Troccaz, J. and Delnondedieu, Y. Semi-active guiding systems in surgery. A two-dof prototype of the passive arm with dynamic constraints (PADyC). Mechatronics, 1996, 6(4), 399-421.

2 Book, W., Charles, R., Davis H. and Gomes, M. The concept and implementation of a passive trajectory enhancing robot. Proc. ASME Dynamics and Controls Div., 1996, 58, 633-638.

3 Moore, C. A., Peshkin, M. A. and Colgate, J. E. A three revolute cobot using CVTs in parallel. In Proceedings of the ASME International Mechanical Engineering Congress and Exposition (IMECE), Nashville, Tennessee, 14-19 November 1999.

4 Reedman, A. V. C. and Bouazza-Marouf, K. Control of an actively constrained robotic joint for passive deployment applications. Proc. Instn Mech. Engrs, Part K: J. Multi-body Dynamics, 2001, 215(K4), 187-197. 